International Journal of Environment, Agriculture and Biotechnology
Vol-6, Issue-5; Sep-Oct, 2021
J Journal Home Page Available: $\underline{\text { https://ijeab.com/ }}$
Journal DOI: $10.22161 /$ ijeab

\title{
Effect of Ulva lactuca L. Seaweed Biostimulant on Seed germination, Growth, and some Biochemical properties of Vigna radiata $\mathrm{L}$.
}

\author{
Megha Pandya*, Shailesh Mehta
}

Botany Department, Sir P.P. Institute of Science, Maharaja Krishnakumarsinhji Bhavnagar University, Bhavnagar, Gujarat *Corresponding Author

Received: 11 Sep 2021; Received in revised form: 30 Sep 2021; Accepted: 08 Oct 2021; Available online: 12 Oct 2021

(C)2021 The Author(s). Published by Infogain Publication. This is an open access article under the CC BY license (https://creativecommons.org/licenses/by/4.0/).

\begin{abstract}
The effect of green marine biostimulant Ulva lactuca evaluated as Biofertilizer to improve growth of Vigna radiata L. Seeds waspresoaked with different concentration seaweed extract of Ulva lactuca such as $0.2 \%, 0.5 \%, 0.7 \%, 1.0 \%, 1.5 \%, 2.0 \%$ and control (without treatment) in different time period such as $0 h, 1 h, 2 h, 3 h, 4 h, 5 h, 6 h, 12 h$ and $24 h$. In Petri dish, after 5 days plant growth parameter like \% seed germination, root length, shoot length, seedling length, seed vigour index, seed stamina index, No. of lateral roots, wet weight, dry weight, \% moisture contentwere observed. After 40, 50 and 60 days some growth parameter like No. of branches per plant, No. of leaves per plant, total height of the plant, No. of flower per plant, No. of pod per plant, length of the pod, No. of seed per pod and leaf area were observed in field trial. In this study, after 50 days Bio-chemical parameter of chlorophyll-a, chlorophyll-b and total chlorophyll, carotenoid, protein, total soluble sugar and reducing sugar measured in plant material. Better result of plant growth observed in higher concentration such as $1.5 \%$ \& $2.0 \%$ of seaweed extract of U. lactuca in Vigna radiata L. and bio-chemical parameter observed in lower concentration such as $0.2 \%$ and $0.7 \%$ of seaweed extract, respectively. Some biochemical parameter like protein,reducing sugar and carotenoid were decrease the amount of concentration compared to control.
\end{abstract}

Keywords - Bio-chemical parameter, Growth parameters, Ulva lactuca, Vigna radiata.

\section{INTRODUCTION}

India has a huge coastline of more than $7000 \mathrm{~km}$ which possesses a large diversity of marine seaweeds. India, the Gujarat state covers a total coastline of $1600 \mathrm{~km}$, which is the longest among all the states of the country, and it covers almost $21.9 \%$ of the Indian coastline of India. Port Okha coast, which is known for its luxuriant growth of a diverse assemblage of seaweeds on Saurashtra coast, is found to have abundant quantities of seaweeds being drifted and washed ashore every year. Port Okha is located at $22^{\circ} 28^{\prime} \mathrm{N}$ latitude and $69^{\circ} 05^{\prime} \mathrm{E}$ longitude and is situated within the opening of Gulf of Kachchh, has a flat rocky intertidal belt, with many tide pools, gullies and crevices. Marine algae found attached to the bottom on solid substrates such as rocks, pebbles, shells, dead corals and plants. Seaweed has great importance, rich in vitamins, minerals, proteins, carbohydrates, amino acids, lipids etc. Due to the richness of bioactive constituents, it revealed many applications such as antioxidant, anticancer, antimalarial, pharmacological and cosmeceutical, activities. Approximately, 271 genera and 1153 species of marine algae, including forms and varieties have been enumerated till date from the Indian waters. Seaweed extracts are soluble, non-toxic and nonpolluting extracts contribute to the uptake of more nutrients from the soil. Seaweeds extract as organic biostimulant is fast becoming accepted practice in agriculture and horticulture due to its beneficial effects (Battacharyya et al., 2015; du Jardin 2015; Khan et al., 2009). In market, seaweed extracts available for several years as fertilizer additives and beneficial result from their use have been reported (Booth, 1969). The biostimulant present in seaweed extract increase the vegetative growth (Di Filippo-Herrera et al., 2018; Briceno-Dominguez et 
al., 2014; Basher et al., 2012; Demir et al., 2006), the leaf chlorophyll content (Vijayakumar et al., 2018; Castellanos-Barriga et al., 2017; Kalaivanan et al., 2012; Matysiak et al., 2011), the stomata density (Spinelli et al., 2010), photosynthetic rate and the fruit production of the plant (Spinelli et al., 2010; Sivasankari et al., 2006). Also the seaweed extracts increased levels of plant defense enzymes (Hernandez-Herrera et al., 2014; Raghavendra et al., 2007), and reduction of harmful seed microflora (Moller \& Smith, 1999) and faster emergence and seedling vigour in several species including wheat (Kumar \& sahoo, 2011), maize (Farooq et al., 2008), pepper (Sivritepe \& Sivritepe 2008), faba bean (ElSheekh\& El-Saled, 2000), barley (Burchett et al., 1998), lettuce (Moller \& Smith, 1998), green gram (Selvam, Balamurugan, Thinakaran \& Sivakumar, 2013) and table beet (Wilczek\& Ng, 1982). Successful application of pregermination techniques in vegetable crop are remarkable since they increase seed germination, are easy to apply and non-risky as well as being environmental friendly. Seaweed extracts are reported to improve seed germination. Traditional priming procedures include hydropriming (seeds primed with water), osmopriming (soaking seeds in osmotic solutions like polyethylene glycol), halopriming (imbibing seeds in salt solution), thermopriming (management of seed with low or high temperatures), solid matrix priming (the agent solid matrices and water are mixed completely and then seeds are added) and biopriming (hydration by biological compounds). Each treatment has benefits and complications and may have variable effects depending upon the type of test, selection of crop, stage of plant development, method of application concentration/dose and duration of treatments (Ashraf \&Foolad, 2005). One of the most important stages of plant life cycle is seed germination. Accelerating the seed germination is one of the important mechanisms on increasing the survival rate of plants. In addition, increasing seed germination rate subsequently increases the yield. Several studies have described the priming of seeds to enhance the germination rate and equal opportunity of growth thereby reducing the emergence time of many horticultural and agricultural crops (Basra et al., 2002; Lee \& Kim, 1999; Brocklehurst \& Dearman, 1983). Several researchers have used different types of soaking treatments of various crops seeds to increase the rate and uniformity of emergence and better establishment of seedlings (Basra et al., 2005; Ashraf et al., 2003; Bose \& Mishra, 1999). The biopriming technique can also be carried out with macroalgae at low concentrations (parts per million) (Sharma et al., 2014). Muthuraman and Ranganathan (2004) selected six species of marine macro algae viz.,
Caulerpa scalpelliformis, Cladophora vagabunda, Enteromorpha compressa, Halimedamacroloba, Ulva faciata and Chaetomorphaantennina to investigate protein, amino acids, total sugars and lipid contents. Venkatesalu et al (2004).

\section{MATERIAL \& METHOD}

\subsection{Collection of Seaweed:}

The green seaweed sampleUlva lactuca Linnaeus belonging to Chlorophyceae family were collected during 2020-2021 at Okha coast is situated at $22^{\circ} 28^{\prime} 7.3272 \mathrm{~N} \&$ $69^{0} 4$ '11.3664 E in the "Gulf of Kutch" on the northwestern most part of Saurashtra in Gujarat, India. After collection of seaweeds, its washed immediately with seawater to remove other debris, other epiphytes and sand particles. Then it was transferred in laboratory and washed thoroughly with simple water up to 3 to 4 times for removal of extra salts on the surface.

\subsection{Preparation of Seaweed Liquid Fertilizer (SLF)}

The seaweed of Ulva lactuca was shade dried followed by oven drying at $60^{\circ} \mathrm{C}$ for 3-5 hours according to the species. The dried sample was grounded with blender to get fine powder and it was stored for future use. These fine powder $10 \mathrm{~g}$ was weighed on electronic balance, $100 \mathrm{ml}$ distilled water was added. The mixture was incubated for One day (24h). Thereafter, the extract was filtered through What-man No. 1 filter paper (Bhosle et al. 1975). The collected filtrate was stored in refrigerator $\left(0-20^{\circ} \mathrm{C}\right)$. The obtained filtrate was considered as $100 \%$. Six different concentrations of solutions such as $0.2 \%$, $0.5 \%, 0.7 \%, 1.0 \%, 1.5 \%$ and $2.0 \%$ were prepared using this $100 \%$ extract and were used for future study.

\subsection{Collection of Vegetable Seeds:}

The Vigna radiata L. seed collected from Vasundhara Agro Agency, Bhavnagar, Gujarat during 2020-21.

\subsection{Seed Soaking:}

The seeds surface was sterilized with $0.1 \% \mathrm{HgCl}_{2}$ up to 12 minutes and washed help of distilled water. Seeds was presoaked with different concentration seaweed extract of Ulva lactuca such as $0.2 \%, 0.5 \%, 0.7 \%, 1.0 \%, 1.5 \%$, $2.0 \%$ and control (without treatment) in different time period such as $0 \mathrm{~h}, 1 \mathrm{~h}, 2 \mathrm{~h}, 3 \mathrm{~h}, 4 \mathrm{~h}, 5 \mathrm{~h}, 6 \mathrm{~h}, 12 \mathrm{~h}$ and $24 \mathrm{~h}$ at room temperature for better germination and early growth. After 5 days all the vegetative parameter was observed.

\subsection{Seed Germination}

Germination was observed daily over a period of 5 day in Vigna radiata $\mathrm{L}$, with the methods of the Association of Official Seed Analysis (AOSA 2005). Three groups of 10 
seeds were tested for germination per treatment (AOSA 2005). Tested Vigna radiata L. seeds were placed on a filter paper in sterilized $90-\mathrm{mm}$ Petri dishes and then treated with $5 \mathrm{ml}-1$ distilled water (Control) and different concentrations $(0.2,0.5,0.7,1.0,1.5$ and $2.0 \%)$ of Seaweed biostimulant. The plates were incubated at $25 \pm 1^{\circ} \mathrm{C}$ and a photoperiod of $16 \mathrm{~h}$ light/8h dark under laboratory. After 5 days measurement of growth parameter like germination percentage, root length, shoot length, seedling length, seed viguor index, seed stamina index, \% Moisture content, No. of lateral roots.

\subsection{Preparation field for further analysis:}

All healthy and specific time selected Vigna radiata L. seed primed in each concentration of seaweed extract and sawing in enough space in each field. Water is sprinkle after sawing seed for maintaining the moisture content. Seaweed extract applied by soil drench method with different concentration with selected time duration. After 30 days, 40 days and 50 days measurement of growth parameter like No. of branches per plant, No. of leaves per plant, No. of flower per plant, No. of pod per plant, Total height of the plant $(\mathrm{cm})$, No. of seed per pod, pod length $(\mathrm{cm})$, Leaf area $\left(\mathrm{cm}^{2}\right)$.

\subsection{Physicochemical Analysis of Seaweed Extract:}

All Physicochemical parameters were analyzed by Handheld Multiparameter meters - Aquasol Digital (Testing Equipment) Model No. AM-AL-01

\subsection{Biochemical Analysis:}

After 50 days biochemical parameter of chlorophyll-a, chlorophyll-b, total chlorophyll, carotenoidwere observed. Protein was determined by Lowry method, Total Soluble Sugar was determined by Anthrone meyhod, reducing sugar was determined by Nelson-Somogyi’s method.

\section{RESULTS}

\subsection{Germination and growth parameters of Vigna radiata $L$. seedling}

Vigna radiata $\mathrm{L}$. showed $80 \%$ germination in the control and $88.88 \%$ in the $1.0 \%$ and $2.0 \%$ seaweed biostimulant treated seeds. A minimum of $81.11 \%$ germination was observed at $0.2 \%$ seaweed biostimulant when compared to all other concentrations of treatment (Table 1 to 7 ). Germination occurred in all treatments in the $2^{\text {nd }}$ day. The effect of seaweed extract showed significantly highest seed germination than control (Table 1 to 7 ). The maximum seedling length of $18.30 \mathrm{~cm}$ was recorded when the plants applied with $1.5 \%$ of Seaweed biostimulants in 12h time duration (Table 5), and minimum seedling length was recorded in $0.2 \%(13.2 \mathrm{~cm})$ of Seaweed biostimulants in $2 \mathrm{~h}$ time period (Table 1 ). A maximum total plant height of $28 \mathrm{~cm}$ was recorded applied with $2.0 \%(4 \mathrm{~h})$ of seaweed biostimulants and minimum in $1.5 \%(12 \mathrm{~h})(18 \mathrm{~cm})$. Also, the plants applied with $2.0 \%$ (4h) seaweed biostimulants increased the No. of branches, No. of leaves, length of the pod and leaf area. A maximum seed vigour index of 1830 in $1.5 \%$ seaweed biostimulants.

\subsection{Physicochemical properties of seaweed extract}

The values of $\mathrm{P}^{\mathrm{H}}$ in Ulva lactuca $2.0 \%$ (Table 11) extract was slightly neutral and slightly basic in other concentration of seaweed extract. The value of Salt concentration (ppm) and EC ( $\mu \mathrm{s})$ continue increases in all concentration of seaweed extract. The value of ORP and TDS were fluctuating in all concentration of seaweed extract.

Table 1: Effect of Ulva lactuca Seaweed biostimulant (0.2\%) on Vigna radiata L.

\begin{tabular}{|c|c|c|c|c|c|c|c|c|c|c|c|}
\hline $\begin{array}{l}\text { Time } \\
\text { Peri } \\
\text { od }\end{array}$ & $\begin{array}{c}\text { Da } \\
\text { y- } 5\end{array}$ & $\begin{array}{c}\text { Germinat } \\
\text { ion \% } \\
\text { (GP) }\end{array}$ & $\begin{array}{c}\text { Root } \\
\text { lengt } \\
\text { h } \\
\text { (radic } \\
\text { le } \\
\text { lengt } \\
\text { h) } \\
\text { (cm) }\end{array}$ & $\begin{array}{c}\text { Shoot } \\
\text { length(c } \\
\text { m) }\end{array}$ & $\begin{array}{l}\text { Seedli } \\
\text { ng } \\
\text { length } \\
(\mathrm{cm})\end{array}$ & $\begin{array}{c}\text { Seed } \\
\text { Stamina } \\
\text { Index } \\
\text { (SSI) }\end{array}$ & $\begin{array}{c}\text { Seed } \\
\text { Vigour } \\
\text { Index } \\
\text { (SVI) }\end{array}$ & $\begin{array}{c}\text { Freshweigh } \\
\text { t(g) }\end{array}$ & $\begin{array}{c}\text { Dry } \\
\text { weight( } \\
\text { g) }\end{array}$ & $\begin{array}{c}\% \\
\text { Moistu } \\
\text { re } \\
\text { conten } \\
t\end{array}$ & $\begin{array}{c}\text { No. } \\
\text { of } \\
\text { Later } \\
\text { al } \\
\text { roots }\end{array}$ \\
\hline $\mathrm{Oh}$ & 8 & 80 & $\begin{array}{c}5.8 \pm 0 . \\
1\end{array}$ & $6.2 \pm 0.1$ & $\begin{array}{c}12.0 \pm 0 \\
.1\end{array}$ & $\begin{array}{c}9.60 \pm 0.0 \\
12\end{array}$ & $\begin{array}{c}960 \pm 1.2 \\
3\end{array}$ & $0.33 \pm 0.02$ & $\begin{array}{c}0.14 \pm 0 . \\
01\end{array}$ & 57.57 & 9 \\
\hline $1 \mathrm{~h}$ & 9 & 90 & $\begin{array}{c}7.0 \pm 0 . \\
2\end{array}$ & $4.2 \pm 0.1$ & $\begin{array}{c}11.2 \pm 0 \\
.2\end{array}$ & $\begin{array}{c}10.08 \pm 0 . \\
12\end{array}$ & $\begin{array}{c}1008 \pm 12 \\
.2\end{array}$ & $0.26 \pm 0.01$ & $\begin{array}{c}0.08 \pm 0 \\
01\end{array}$ & 69.23 & 25 \\
\hline
\end{tabular}




\begin{tabular}{|c|c|c|c|c|c|c|c|c|c|c|c|}
\hline $2 \mathrm{~h}$ & 10 & 100 & $\begin{array}{c}6.6 \pm 0 . \\
2\end{array}$ & $6.6 \pm 0.1$ & $\begin{array}{c}13.2 \pm 0 \\
.1\end{array}$ & $\begin{array}{c}13.20 \pm 0 \\
23\end{array}$ & $\begin{array}{c}1320 \pm 23 \\
.20\end{array}$ & $0.38 \pm 0.01$ & $\begin{array}{c}0.20 \pm 0 \\
01\end{array}$ & 47.36 & 10 \\
\hline $3 \mathrm{~h}$ & 5 & 50 & $\begin{array}{c}6.2 \pm 0 \\
1\end{array}$ & $9.0 \pm 0.1$ & $\begin{array}{c}15.2 \pm 0 \\
.1\end{array}$ & $\begin{array}{c}7.60 \pm 0.0 \\
13\end{array}$ & $\begin{array}{c}760 \pm 1.3 \\
5\end{array}$ & $0.37 \pm 0.02$ & $\begin{array}{c}0.22 \pm 0 \\
02\end{array}$ & 40.54 & 20 \\
\hline $4 h$ & 9 & 90 & $\begin{array}{c}4.9 \pm 0 . \\
1\end{array}$ & $6.4 \pm 0.2$ & $\begin{array}{c}11.3 \pm 0 \\
.2\end{array}$ & $\begin{array}{c}10.17 \pm 0 . \\
23\end{array}$ & $\begin{array}{c}1017 \pm 23 \\
.12\end{array}$ & $0.38 \pm 0.01$ & $\begin{array}{c}0.21 \pm 0 \\
01\end{array}$ & 44.73 & 11 \\
\hline $5 \mathrm{~h}$ & 9 & 90 & $\begin{array}{c}7.3 \pm 0 . \\
2\end{array}$ & $6.0 \pm 0.3$ & $\begin{array}{c}13.3 \pm 0 \\
.2\end{array}$ & $\begin{array}{c}11.97 \pm 0 \\
24\end{array}$ & $\begin{array}{c}1197 \pm 24 \\
.61\end{array}$ & $0.36 \pm 0.01$ & $\begin{array}{c}0.20 \pm 0 . \\
01\end{array}$ & 44.44 & 20 \\
\hline $6 \mathrm{~h}$ & 9 & 90 & $\begin{array}{c}4.4 \pm 0 . \\
1\end{array}$ & $8.0 \pm 0.1$ & $\begin{array}{c}12.4 \pm 0 \\
.1\end{array}$ & $\begin{array}{c}11.16 \pm 0 . \\
24\end{array}$ & $\begin{array}{c}1116 \pm 24 \\
.11\end{array}$ & $0.35 \pm 0.02$ & $\begin{array}{c}0.18 \pm 0 \\
02\end{array}$ & 48.57 & 16 \\
\hline $12 \mathrm{~h}$ & 6 & 60 & $\begin{array}{c}5.0 \pm 0 . \\
1\end{array}$ & $6.0 \pm 0.2$ & $\begin{array}{c}11.0 \pm 0 \\
.2\end{array}$ & $\begin{array}{c}6.60 \pm 0.0 \\
12\end{array}$ & $\begin{array}{c}660 \pm 1.2 \\
3\end{array}$ & $0.08 \pm 0.01$ & $\begin{array}{c}0.02 \pm 0 . \\
01\end{array}$ & 75.00 & 12 \\
\hline $24 \mathrm{~h}$ & 8 & 80 & $\begin{array}{c}3.2 \pm 0 . \\
2\end{array}$ & $10.0 \pm 0.1$ & $\begin{array}{c}13.2 \pm 0 \\
.1\end{array}$ & $\begin{array}{c}10.56 \pm 0 . \\
22\end{array}$ & $\begin{array}{c}1056 \pm 22 \\
.10\end{array}$ & $0.43 \pm 0.01$ & $\begin{array}{c}0.18 \pm 0 \\
01\end{array}$ & 58.13 & 18 \\
\hline
\end{tabular}

Table 2: Effect of Ulva lactuca Seaweed biostimulant (0.5\%) on Vigna radiata L.

\begin{tabular}{|c|c|c|c|c|c|c|c|c|c|c|c|}
\hline $\begin{array}{l}\text { Tim } \\
\text { e } \\
\text { Peri } \\
\text { od }\end{array}$ & $\begin{array}{c}\text { Da } \\
\text { y- } 5\end{array}$ & $\begin{array}{c}\text { Germinat } \\
\text { ion \% } \\
\text { (GP) }\end{array}$ & $\begin{array}{c}\text { Root } \\
\text { length(rad } \\
\text { icle } \\
\text { length) } \\
\text { (cm) }\end{array}$ & $\begin{array}{l}\text { Shoot } \\
\text { length(c } \\
\text { m) }\end{array}$ & $\begin{array}{l}\text { Seedli } \\
\text { ng } \\
\text { length } \\
(\mathrm{cm})\end{array}$ & $\begin{array}{c}\text { Seed } \\
\text { Stamin } \\
\text { a Index } \\
\text { (SSI) }\end{array}$ & $\begin{array}{c}\text { Seed } \\
\text { Vigour } \\
\text { Index } \\
\text { (SVI) }\end{array}$ & $\begin{array}{c}\text { Fresh } \\
\text { weight } \\
\text { (g) }\end{array}$ & $\begin{array}{c}\text { Dry } \\
\text { weight( } \\
\text { g) }\end{array}$ & $\begin{array}{c}\% \\
\text { Moistu } \\
\text { re } \\
\text { conten } \\
\text { t }\end{array}$ & $\begin{array}{c}\begin{array}{c}\text { No. } \\
\text { of } \\
\text { Later }\end{array} \\
\text { al } \\
\text { roots }\end{array}$ \\
\hline $\mathbf{0 h}$ & 9 & 90 & $3.4 \pm 0.1$ & $7.2 \pm 0.1$ & $\begin{array}{c}10.6 \pm 0 \\
.1\end{array}$ & $\begin{array}{c}9.54 \pm 0 \\
012\end{array}$ & $\begin{array}{c}954 \pm 1.2 \\
3\end{array}$ & $\begin{array}{c}0.22 \pm 0 \\
01\end{array}$ & $\begin{array}{c}0.08 \pm 0 \\
01\end{array}$ & 63.63 & 6 \\
\hline 1h & 9 & 90 & $7.6 \pm 0.1$ & $4.2 \pm 0.2$ & $\begin{array}{c}11.8 \pm 0 \\
.1\end{array}$ & $\begin{array}{c}10.62 \pm 0 \\
.12\end{array}$ & $\begin{array}{c}1062 \pm 12 \\
.21\end{array}$ & $\begin{array}{c}0.36 \pm 0 \\
02\end{array}$ & $\begin{array}{c}0.10 \pm 0 \\
02\end{array}$ & 72.22 & 21 \\
\hline $2 \mathrm{~h}$ & 10 & 100 & $9.0 \pm 0.2$ & $5.0 \pm 0.2$ & $\begin{array}{c}14.0 \pm 0 \\
.2\end{array}$ & $\begin{array}{c}14.00 \pm 0 \\
.23\end{array}$ & $\begin{array}{c}1400 \pm 23 \\
.24\end{array}$ & $\begin{array}{c}0.39 \pm 0 \\
03\end{array}$ & $\begin{array}{c}0.12 \pm 0 \\
01\end{array}$ & 69.23 & 15 \\
\hline $3 h$ & 8 & 80 & $6.4 \pm 0.1$ & $2.9 \pm 0.1$ & $\begin{array}{c}9.3 \pm 0 . \\
1\end{array}$ & $\begin{array}{c}7.44 \pm 0 \\
023\end{array}$ & $\begin{array}{c}744 \pm 2.3 \\
1\end{array}$ & $\begin{array}{c}0.18 \pm 0 \\
04\end{array}$ & $\begin{array}{c}0.06 \pm 0 \\
01\end{array}$ & 66.66 & 14 \\
\hline $4 h$ & 9 & 90 & $5.2 \pm 0.1$ & $4.0 \pm 0.1$ & $\begin{array}{c}9.2 \pm 0 . \\
1\end{array}$ & $\begin{array}{c}8.28 \pm 0 \\
031\end{array}$ & $\begin{array}{c}828 \pm 3.1 \\
1\end{array}$ & $\begin{array}{c}0.29 \pm 0 \\
01\end{array}$ & $\begin{array}{c}0.10 \pm 0 \\
01\end{array}$ & 65.51 & 20 \\
\hline $5 \mathrm{~h}$ & 8 & 80 & $2.2 \pm 0.2$ & $2.0 \pm 0.1$ & $\begin{array}{c}4.2 \pm 0 . \\
2\end{array}$ & $\begin{array}{c}3.36 \pm 0 \\
011\end{array}$ & $\begin{array}{c}336 \pm 1.1 \\
0\end{array}$ & $\begin{array}{c}0.29 \pm 0 \\
03\end{array}$ & $\begin{array}{c}0.09 \pm 0 \\
006\end{array}$ & 68.96 & 13 \\
\hline $6 h$ & 8 & 80 & $6.2 \pm 0.1$ & $5.4 \pm 0.2$ & $\begin{array}{c}11.6 \pm 0 \\
.1\end{array}$ & $\begin{array}{c}9.28 \pm 0 \\
010\end{array}$ & $\begin{array}{c}928 \pm 1.0 \\
9\end{array}$ & $\begin{array}{c}0.25 \pm 0 \\
02\end{array}$ & $\begin{array}{c}0.08 \pm 0 \\
001\end{array}$ & 68.00 & 20 \\
\hline $12 \mathrm{~h}$ & 7 & 70 & $4.2 \pm 0.2$ & $13.0 \pm 0.1$ & $\begin{array}{c}17.2 \pm 0 \\
.2\end{array}$ & $\begin{array}{c}12.04 \pm 0 \\
.25\end{array}$ & $\begin{array}{c}1204 \pm 25 \\
.12\end{array}$ & $\begin{array}{c}0.51 \pm 0 \\
03\end{array}$ & $\begin{array}{c}0.23 \pm 0 \\
02\end{array}$ & 54.90 & 18 \\
\hline $24 h$ & 8 & 80 & $5.9 \pm 0.1$ & $7.2 \pm 0.1$ & $\begin{array}{c}13.1 \pm 0 \\
.1\end{array}$ & 10.48 & 1048 & 0.33 & 0.11 & 66.66 & 15 \\
\hline
\end{tabular}

$($ Result $=$ Mean \pm std $)$ 
Table 3: Effect of Ulva lactuca Seaweed biostimulant (0.7\%) on Vigna radiata L.

\begin{tabular}{|c|c|c|c|c|c|c|c|c|c|c|c|}
\hline $\begin{array}{l}\text { Time } \\
\text { Peri } \\
\text { od }\end{array}$ & $\begin{array}{c}\text { Da } \\
\text { y- } 5\end{array}$ & $\begin{array}{c}\text { Germinat } \\
\text { ion \% } \\
\text { (GP) }\end{array}$ & $\begin{array}{c}\text { Root } \\
\text { length(rad } \\
\text { icle } \\
\text { length) } \\
\text { (cm) }\end{array}$ & $\begin{array}{l}\text { Shoot } \\
\text { length(c } \\
\text { m) }\end{array}$ & $\begin{array}{l}\text { Seedli } \\
\text { ng } \\
\text { length } \\
(\mathrm{cm})\end{array}$ & $\begin{array}{c}\text { Seed } \\
\text { Stamina } \\
\text { Index } \\
\text { (SSI) }\end{array}$ & $\begin{array}{c}\text { Seed } \\
\text { Vigour } \\
\text { Index } \\
\text { (SVI) }\end{array}$ & $\begin{array}{c}\text { Wet } \\
\text { weight( } \\
\text { g) }\end{array}$ & $\begin{array}{c}\text { Dry } \\
\text { weight( } \\
\text { g) }\end{array}$ & $\begin{array}{c}\% \\
\text { Moistu } \\
\text { re } \\
\text { conten } \\
\mathbf{t}\end{array}$ & $\begin{array}{c}\text { No. } \\
\text { of } \\
\text { Later } \\
\text { al } \\
\text { roots }\end{array}$ \\
\hline $\mathbf{0 h}$ & 8 & 80 & $6.6 \pm 0.1$ & $7.2 \pm 0.1$ & $\begin{array}{c}13.8 \pm 0 \\
.1\end{array}$ & $\begin{array}{c}11.04 \pm 0 \\
12\end{array}$ & $\begin{array}{c}1104 \pm 12 \\
.23\end{array}$ & $\begin{array}{c}0.33 \pm 0 \\
01\end{array}$ & $\begin{array}{c}0.12 \pm 0 \\
01\end{array}$ & 63.63 & 15 \\
\hline $1 \mathrm{~h}$ & 7 & 70 & $5.2 \pm 0.1$ & $4.2 \pm 0.1$ & $\begin{array}{c}9.4 \pm 0 . \\
1\end{array}$ & $\begin{array}{c}6.58 \pm 0.0 \\
21\end{array}$ & $\begin{array}{c}658 \pm 2.1 \\
4\end{array}$ & $\begin{array}{c}0.24 \pm 0 \\
02\end{array}$ & $\begin{array}{c}0.09 \pm 0 \\
01\end{array}$ & 62.50 & 15 \\
\hline $2 \mathrm{~h}$ & 8 & 80 & $7.8 \pm 0.2$ & $10.6 \pm 0.2$ & $\begin{array}{c}18.4 \pm 0 \\
.2\end{array}$ & $\begin{array}{c}14.72 \pm 0 \\
21\end{array}$ & $\begin{array}{c}1472 \pm 21 \\
.14\end{array}$ & $\begin{array}{c}0.66 \pm 0 \\
01\end{array}$ & $\begin{array}{c}0.33 \pm 0 \\
02\end{array}$ & 50.00 & 16 \\
\hline $3 h$ & 8 & 80 & $7.8 \pm 0.1$ & $5.4 \pm 0.2$ & $\begin{array}{c}13.2 \pm 0 \\
.1\end{array}$ & $\begin{array}{c}10.56 \pm 0 \\
22\end{array}$ & $\begin{array}{c}1056 \pm 22 \\
.31\end{array}$ & $\begin{array}{c}0.32 \pm 0 \\
01\end{array}$ & $\begin{array}{c}0.15 \pm 0 \\
01\end{array}$ & 53.12 & 6 \\
\hline $4 h$ & 8 & 80 & $6.5 \pm 0.1$ & $4.0 \pm 0.2$ & $\begin{array}{c}10.5 \pm 0 \\
.1\end{array}$ & $\begin{array}{c}8.40 \pm 0.0 \\
12\end{array}$ & $\begin{array}{c}840 \pm 1.2 \\
3\end{array}$ & $\begin{array}{c}0.31 \pm 0 \\
01\end{array}$ & $\begin{array}{c}0.12 \pm 0 \\
02\end{array}$ & 61.29 & 14 \\
\hline $5 \mathrm{~h}$ & 9 & 90 & $6.5 \pm 0.1$ & $6.0 \pm 0.1$ & $\begin{array}{c}12.5 \pm 0 \\
.2\end{array}$ & $\begin{array}{c}11.25 \pm 0 \\
23\end{array}$ & $\begin{array}{c}1125 \pm 23 \\
.11\end{array}$ & $\begin{array}{c}0.31 \pm 0 \\
01\end{array}$ & $\begin{array}{c}0.14 \pm 0 \\
01\end{array}$ & 54.83 & 18 \\
\hline $6 h$ & 9 & 90 & $6.0 \pm 0.2$ & $10.0 \pm 0.1$ & $\begin{array}{c}16.0 \pm 0 \\
.2\end{array}$ & $\begin{array}{c}14.40 \pm 0 \\
11\end{array}$ & $\begin{array}{c}1440 \pm 11 \\
.56\end{array}$ & $\begin{array}{c}0.34 \pm 0 \\
01\end{array}$ & $\begin{array}{c}0.13 \pm 0 \\
01\end{array}$ & 61.76 & 17 \\
\hline $12 \mathrm{~h}$ & 9 & 90 & $6.2 \pm 0.2$ & $6.0 \pm 0.1$ & $\begin{array}{c}12.2 \pm 0 \\
.1\end{array}$ & $\begin{array}{c}10.98 \pm 0 \\
32\end{array}$ & $\begin{array}{c}1098 \pm 32 \\
.08\end{array}$ & $\begin{array}{c}0.30 \pm 0 \\
02\end{array}$ & $\begin{array}{c}0.11 \pm 0 . \\
01\end{array}$ & 63.33 & 15 \\
\hline $24 h$ & 9 & 90 & $7.0 \pm 0.1$ & $6.0 \pm 0.2$ & $\begin{array}{c}13.0 \pm 0 \\
.1\end{array}$ & $\begin{array}{c}11.70 \pm 0 \\
24\end{array}$ & $\begin{array}{c}1170 \pm 24 \\
.10\end{array}$ & $\begin{array}{c}0.22 \pm 0 \\
02\end{array}$ & $\begin{array}{c}0.09 \pm 0 \\
01\end{array}$ & 59.09 & 22 \\
\hline
\end{tabular}

$($ Result $=$ Mean \pm std $)$

Table 4: Effect of Ulva lactuca Seaweed biostimulant (1.0\%) on Vigna radiata L.

\begin{tabular}{|c|c|c|c|c|c|c|c|c|c|c|c|}
\hline $\begin{array}{l}\text { Time } \\
\text { Peri } \\
\text { od }\end{array}$ & $\begin{array}{c}\text { Da } \\
y-5\end{array}$ & $\begin{array}{c}\text { Germinat } \\
\text { ion \% } \\
\text { (GP) }\end{array}$ & $\begin{array}{c}\text { Root } \\
\text { length(rad } \\
\text { icle } \\
\text { length) } \\
\text { (cm) }\end{array}$ & $\begin{array}{c}\text { Shoot } \\
\text { length(c } \\
\text { m) }\end{array}$ & $\begin{array}{l}\text { Seedli } \\
\text { ng } \\
\text { length } \\
\text { (cm) }\end{array}$ & $\begin{array}{c}\text { Seed } \\
\text { Stamina } \\
\text { Index } \\
\text { (SSI) }\end{array}$ & $\begin{array}{c}\text { Seed } \\
\text { Vigour } \\
\text { Index } \\
\text { (SVI) }\end{array}$ & $\begin{array}{c}\text { Wet } \\
\text { weight( } \\
\text { g) }\end{array}$ & $\begin{array}{c}\text { Dry } \\
\text { weight( } \\
\text { g) }\end{array}$ & $\begin{array}{c}\% \\
\text { Moistu } \\
\text { re } \\
\text { conten } \\
\mathbf{t}\end{array}$ & $\begin{array}{c}\text { No. } \\
\text { of } \\
\text { Later } \\
\text { al } \\
\text { roots }\end{array}$ \\
\hline $\mathbf{0 h}$ & 10 & 100 & $5.4 \pm 0.1$ & $4.4 \pm 0.1$ & $\begin{array}{c}9.8 \pm 0 . \\
1\end{array}$ & $\begin{array}{c}0.98 \pm 0.0 \\
12\end{array}$ & $\begin{array}{c}980 \pm 1.2 \\
3\end{array}$ & $\begin{array}{c}0.12 \pm 0 \\
01\end{array}$ & $\begin{array}{c}0.06 \pm 0 \\
01\end{array}$ & 50.00 & 12 \\
\hline 1h & 9 & 90 & $4.0 \pm 0.1$ & $7.4 \pm 0.1$ & $\begin{array}{c}11.4 \pm 0 \\
.1\end{array}$ & $\begin{array}{c}10.26 \pm 0 \\
25\end{array}$ & $\begin{array}{c}1026 \pm 25 \\
.10\end{array}$ & $\begin{array}{c}0.24 \pm 0 \\
02\end{array}$ & $\begin{array}{c}0.09 \pm 0 \\
01\end{array}$ & 62.50 & 15 \\
\hline $2 h$ & 9 & 90 & $6.6 \pm 0.2$ & $10.5 \pm 0.1$ & $\begin{array}{c}17.1 \pm 0 \\
.2\end{array}$ & $\begin{array}{c}15.39 \pm 0 \\
36\end{array}$ & $\begin{array}{c}1539 \pm 36 \\
.21\end{array}$ & $\begin{array}{c}0.42 \pm 0 \\
01\end{array}$ & $\begin{array}{c}0.21 \pm 0 \\
01\end{array}$ & 50.00 & 16 \\
\hline 3h & 8 & 80 & $6.2 \pm 0.1$ & $11.0 \pm 0.2$ & $\begin{array}{c}17.2 \pm 0 \\
.1\end{array}$ & $\begin{array}{c}13.76 \pm 0 \\
25\end{array}$ & $\begin{array}{c}1376 \pm 25 \\
.10\end{array}$ & $\begin{array}{c}0.45 \pm 0 \\
01\end{array}$ & $\begin{array}{c}0.22 \pm 0 \\
01\end{array}$ & 51.11 & 13 \\
\hline $4 h$ & 9 & 90 & $6.6 \pm 0.1$ & $9.1 \pm 0.2$ & $\begin{array}{c}15.7 \pm 0 \\
.1\end{array}$ & $\begin{array}{c}14.13 \pm 0 . \\
26\end{array}$ & $\begin{array}{c}1413 \pm 26 \\
.12\end{array}$ & $\begin{array}{c}0.35 \pm 0 \\
01\end{array}$ & $\begin{array}{c}0.16 \pm 0 \\
01\end{array}$ & 54.28 & 10 \\
\hline $5 \mathrm{~h}$ & 9 & 90 & $5.8 \pm 0.2$ & $7.6 \pm 0.1$ & $\begin{array}{c}13.4 \pm 0 \\
1\end{array}$ & $\begin{array}{c}12.06 \pm 0 \\
22\end{array}$ & $\begin{array}{c}1206 \pm 22 \\
.21\end{array}$ & $\begin{array}{c}0.20 \pm 0 \\
02\end{array}$ & $\begin{array}{c}0.08 \pm 0 \\
02\end{array}$ & 60.00 & 16 \\
\hline
\end{tabular}




\begin{tabular}{|c|c|c|c|c|c|c|c|c|c|c|c|}
\hline 6h & 8 & 80 & $5.3 \pm 0.1$ & $5.2 \pm 0.1$ & $10.5 \pm 0$ & $8.40 \pm 0.0$ & $840 \pm 1.1$ & $0.27 \pm 0$. & $0.12 \pm 0$. & 55.55 & 15 \\
& & & & .2 & 11 & 2 & 02 & 01 & \\
\hline $\mathbf{1 2 h}$ & 9 & 90 & $8.0 \pm 0.2$ & $9.2 \pm 0.1$ & $17.2 \pm 0$ & $15.48 \pm 0$. & $1548 \pm 28$ & $0.48 \pm 0$. & $0.24 \pm 0$. & 50.00 & 13 \\
& & & & .1 & 28 & .21 & 02 & 01 & \\
\hline $\mathbf{2 4 h}$ & 9 & 90 & $4.0 \pm 0.1$ & $4.6 \pm 0.1$ & $8.6 \pm 0$. & $7.74 \pm 0.0$ & $774 \pm 1.0$ & $0.17 \pm 0$. & $0.08 \pm 0$. & 52.94 \\
\end{tabular}

(Result=Mean \pm std $)$

Table 5: Effect of Ulva lactuca Seaweed biostimulant (1.5\%) on Vigna radiata L.

\begin{tabular}{|c|c|c|c|c|c|c|c|c|c|c|c|}
\hline $\begin{array}{l}\text { Time } \\
\text { Peri } \\
\text { od }\end{array}$ & $\begin{array}{l}\text { Da } \\
y-5\end{array}$ & $\begin{array}{c}\text { Germinat } \\
\text { ion \% } \\
\text { (GP) }\end{array}$ & $\begin{array}{c}\text { Root } \\
\text { length(rad } \\
\text { icle } \\
\text { length) } \\
\text { (cm) }\end{array}$ & $\begin{array}{c}\text { Shoot } \\
\text { length(c } \\
\text { m) }\end{array}$ & $\begin{array}{l}\text { Seedli } \\
\text { ng } \\
\text { length } \\
(\mathrm{cm})\end{array}$ & $\begin{array}{c}\text { Seed } \\
\text { Stamina } \\
\text { Index } \\
\text { (SSI) }\end{array}$ & $\begin{array}{l}\text { Seed } \\
\text { Vigour } \\
\text { Index } \\
\text { (SVI) }\end{array}$ & $\begin{array}{c}\text { Wet } \\
\text { weight( } \\
\text { g) }\end{array}$ & $\begin{array}{c}\text { Dry } \\
\text { weight( } \\
\text { g) }\end{array}$ & $\begin{array}{c}\% \\
\text { Moistu } \\
\text { re } \\
\text { conten } \\
\text { t }\end{array}$ & $\begin{array}{c}\text { No. } \\
\text { of } \\
\text { Later } \\
\text { al } \\
\text { roots }\end{array}$ \\
\hline $\mathbf{O h}$ & 10 & 100 & $4.6 \pm 0.1$ & $3.0 \pm 0.1$ & $\begin{array}{c}7.6 \pm 0 . \\
1\end{array}$ & $\begin{array}{c}7.60 \pm 0.0 \\
12\end{array}$ & $\begin{array}{c}760 \pm 1.2 \\
3\end{array}$ & $\begin{array}{c}0.13 \pm 0 . \\
02\end{array}$ & $\begin{array}{c}0.03 \pm 0 \\
01\end{array}$ & 76.92 & 13 \\
\hline 1h & 9 & 90 & $4.2 \pm 0.1$ & $1.4 \pm 0.1$ & $\begin{array}{c}5.6 \pm 0 . \\
1\end{array}$ & $\begin{array}{c}5.04 \pm 0.0 \\
32\end{array}$ & $\begin{array}{c}504 \pm 3.2 \\
1\end{array}$ & $\begin{array}{c}0.42 \pm 0 \\
01\end{array}$ & $\begin{array}{c}0.21 \pm 0 \\
01\end{array}$ & 50.00 & 8 \\
\hline $2 h$ & 9 & 90 & $4.0 \pm 0.1$ & $3.8 \pm 0.1$ & $\begin{array}{c}7.8 \pm 0 . \\
1\end{array}$ & $\begin{array}{c}7.02 \pm 0.0 \\
24\end{array}$ & $\begin{array}{c}702 \pm 2.4 \\
1\end{array}$ & $\begin{array}{c}0.09 \pm 0 \\
01\end{array}$ & $\begin{array}{c}0.03 \pm 0 \\
01\end{array}$ & 66.66 & 9 \\
\hline 3h & 10 & 100 & $3.9 \pm 0.2$ & $9.0 \pm 0.2$ & $\begin{array}{c}12.9 \pm 0 \\
.2\end{array}$ & $\begin{array}{c}12.90 \pm 0 \\
15\end{array}$ & $\begin{array}{c}1290 \pm 15 \\
.23\end{array}$ & $\begin{array}{c}0.29 \pm 0 \\
02\end{array}$ & $\begin{array}{c}0.12 \pm 0 \\
01\end{array}$ & 58.62 & 8 \\
\hline $4 h$ & 9 & 90 & $6.2 \pm 0.1$ & $6.4 \pm 0.2$ & $\begin{array}{c}12.6 \pm 0 \\
.1\end{array}$ & $\begin{array}{c}11.34 \pm 0 \\
23\end{array}$ & $\begin{array}{c}1134 \pm 23 \\
.11\end{array}$ & $\begin{array}{c}0.27 \pm 0 \\
02\end{array}$ & $\begin{array}{c}0.14 \pm 0 \\
01\end{array}$ & 48.14 & 9 \\
\hline $5 h$ & 8 & 80 & $3.9 \pm 0.2$ & $8.2 \pm 0.1$ & $\begin{array}{c}12.1 \pm 0 \\
.2\end{array}$ & $\begin{array}{c}9.68 \pm 0.0 \\
31\end{array}$ & $\begin{array}{c}968 \pm 3.1 \\
0\end{array}$ & $\begin{array}{c}0.26 \pm 0 \\
01\end{array}$ & $\begin{array}{c}0.09 \pm 0 \\
01\end{array}$ & 65.38 & 16 \\
\hline $6 \mathrm{~h}$ & 6 & 60 & $7.5 \pm 0.1$ & $11.0 \pm 0.1$ & $\begin{array}{c}18.5 \pm 0 \\
.1\end{array}$ & $\begin{array}{c}11.10 \pm 0 \\
25\end{array}$ & $\begin{array}{c}1110 \pm 25 \\
.09\end{array}$ & $\begin{array}{c}0.28 \pm 0 \\
01\end{array}$ & $\begin{array}{c}0.10 \pm 0 \\
01\end{array}$ & 64.28 & 19 \\
\hline $12 \mathrm{~h}$ & 10 & 100 & $7.5 \pm 0.1$ & $10.8 \pm 0.1$ & $\begin{array}{c}18.3 \pm 0 \\
.2\end{array}$ & $\begin{array}{c}18.30 \pm 0 \\
12\end{array}$ & $\begin{array}{c}1830 \pm 12 \\
.21\end{array}$ & $\begin{array}{c}0.38 \pm 0 \\
02\end{array}$ & $\begin{array}{c}0.16 \pm 0 \\
01\end{array}$ & 57.89 & 19 \\
\hline $24 h$ & 8 & 80 & $6.3 \pm 0.1$ & $8.0 \pm 0.1$ & $\begin{array}{c}14.3 \pm 0 \\
.1\end{array}$ & $\begin{array}{c}11.44 \pm 0 \\
20\end{array}$ & $\begin{array}{c}1144 \pm 20 \\
.08\end{array}$ & $\begin{array}{c}0.22 \pm 0 \\
01\end{array}$ & $\begin{array}{c}0.10 \pm 0 \\
01\end{array}$ & 54.54 & 15 \\
\hline
\end{tabular}

(Result=Mean \pm std $)$

Table 6: Effect of Ulva lactuca Seaweed biostimulants (2.0\%) on Vigna radiata L.

\begin{tabular}{|c|c|c|c|c|c|c|c|c|c|c|c|}
\hline $\begin{array}{l}\text { Tim } \\
\text { e } \\
\text { Peri } \\
\text { od }\end{array}$ & $\begin{array}{c}\text { Da } \\
\text { y- } \\
5\end{array}$ & $\begin{array}{c}\text { Germinat } \\
\text { ion \% } \\
\text { (GP) }\end{array}$ & $\begin{array}{c}\text { Root } \\
\text { length(rad } \\
\text { icle } \\
\text { length) } \\
\text { (cm) }\end{array}$ & $\begin{array}{c}\text { Shoot } \\
\text { length(c } \\
\text { m) }\end{array}$ & $\begin{array}{c}\text { Seedli } \\
\text { ng } \\
\text { length } \\
(\mathrm{cm})\end{array}$ & $\begin{array}{c}\text { Seed } \\
\text { Stamin } \\
\text { a Index } \\
\text { (SSI) }\end{array}$ & $\begin{array}{c}\text { Seed } \\
\text { Vigour } \\
\text { Index } \\
\text { (SVI) }\end{array}$ & $\begin{array}{c}\text { Wet } \\
\text { weight } \\
\text { (g) }\end{array}$ & $\begin{array}{c}\text { Dry } \\
\text { weight(g } \\
\text { ) }\end{array}$ & $\begin{array}{c}\% \\
\text { Moist } \\
\text { ure } \\
\text { conten } \\
t\end{array}$ & $\begin{array}{c}\text { No. } \\
\text { of } \\
\text { Later } \\
\text { al } \\
\text { roots }\end{array}$ \\
\hline $\mathbf{0 h}$ & 7 & 70 & $5.7 \pm 0.1$ & $6.8 \pm 0.1$ & $\begin{array}{c}12.5 \pm \\
0.1\end{array}$ & $\begin{array}{c}8.75 \pm 0 \\
023\end{array}$ & $\begin{array}{c}875 \pm 2.3 \\
1\end{array}$ & $\begin{array}{c}0.27 \pm 0 \\
01\end{array}$ & $0.1 \pm 0.01$ & 62.96 & 7 \\
\hline $1 \mathrm{~h}$ & 8 & 80 & $4.2 \pm 0.1$ & $2.8 \pm 0.1$ & $\begin{array}{c}7.0 \pm 0 . \\
1\end{array}$ & $\begin{array}{c}5.60 \pm 0 \\
052\end{array}$ & $\begin{array}{c}560 \pm 5.2 \\
1\end{array}$ & $\begin{array}{c}0.1 \pm 0.0 \\
1\end{array}$ & $\begin{array}{c}0.04 \pm 00 \\
2\end{array}$ & 60.0 & 10 \\
\hline
\end{tabular}




\begin{tabular}{|c|c|c|c|c|c|c|c|c|c|c|c|}
\hline $2 \mathrm{~h}$ & 10 & 100 & $5.8 \pm 0.2$ & $8.2 \pm 0.2$ & $\begin{array}{c}14.0 \pm \\
0.1\end{array}$ & $\begin{array}{c}14.00 \pm 0 \\
.32\end{array}$ & $\begin{array}{c}1400 \pm 32 \\
.10\end{array}$ & $\begin{array}{c}0.3 \pm 0.0 \\
2\end{array}$ & $0.01 \pm 0.1$ & 96.66 & 17 \\
\hline $3 h$ & 9 & 90 & $4.1 \pm 0.2$ & $5.2 \pm 0.1$ & $\begin{array}{c}9.3 \pm 0 . \\
2\end{array}$ & $\begin{array}{c}8.37 \pm 0 \\
041\end{array}$ & $\begin{array}{c}837 \pm 4.1 \\
1\end{array}$ & $\begin{array}{c}0.27 \pm 0 \\
01\end{array}$ & $0.1 \pm 0.01$ & 62.96 & 8 \\
\hline $4 h$ & 9 & 90 & $5.4 \pm 0.1$ & $6.6 \pm 0.1$ & $\begin{array}{c}12.0 \pm \\
0.1\end{array}$ & $\begin{array}{c}10.80 \pm 0 \\
.25\end{array}$ & $\begin{array}{c}1080 \pm 25 \\
.56\end{array}$ & $\begin{array}{c}0.24 \pm 0 \\
02\end{array}$ & $0.1 \pm 0.01$ & 58.33 & 18 \\
\hline $5 \mathrm{~h}$ & 6 & 60 & $6.2 \pm 0.1$ & $4.0 \pm 0.2$ & $\begin{array}{c}10.2 \pm \\
0.1\end{array}$ & $\begin{array}{c}6.12 \pm 0 . \\
061\end{array}$ & $\begin{array}{c}612 \pm 6.1 \\
0\end{array}$ & $\begin{array}{c}0.09 \pm 0 \\
02\end{array}$ & $\begin{array}{c}0.006 \pm 0 . \\
001\end{array}$ & 93.33 & 15 \\
\hline $6 \mathrm{~h}$ & 8 & 80 & $5.6 \pm 0.2$ & $2.4 \pm 0.2$ & $\begin{array}{c}8.0 \pm 0 . \\
2\end{array}$ & $\begin{array}{c}6.40 \pm 0 \\
071\end{array}$ & $\begin{array}{c}640 \pm 7.1 \\
2\end{array}$ & $\begin{array}{c}0.2 \pm 0.0 \\
1\end{array}$ & $\begin{array}{c}0.08 \pm 0.0 \\
1\end{array}$ & 60.0 & 11 \\
\hline $12 \mathrm{~h}$ & 9 & 90 & $4.6 \pm 0.1$ & $5.0 \pm 0.2$ & $\begin{array}{c}9.6 \pm 0 . \\
2\end{array}$ & $\begin{array}{c}8.64 \pm 0 . \\
091\end{array}$ & $\begin{array}{c}864 \pm 9.1 \\
2\end{array}$ & $\begin{array}{c}0.17 \pm 0 \\
01\end{array}$ & $\begin{array}{c}0.09 \pm 0.0 \\
1\end{array}$ & 47.05 & 9 \\
\hline $24 h$ & 6 & 60 & $5.2 \pm 0.1$ & $2.4 \pm 0.1$ & $\begin{array}{c}7.6 \pm 0 . \\
1\end{array}$ & $\begin{array}{c}4.56 \pm 0 \\
051\end{array}$ & $\begin{array}{c}456 \pm 5.1 \\
4\end{array}$ & $\begin{array}{c}0.16 \pm 0 \\
01\end{array}$ & $\begin{array}{c}0.06 \pm 0.0 \\
1\end{array}$ & 62.5 & 7 \\
\hline
\end{tabular}

(Result=Mean \pm std)

Table 7: Control plant on Vigna radiata $L$.

\begin{tabular}{|c|c|c|c|c|c|c|c|c|c|c|c|}
\hline $\begin{array}{c}\text { Time } \\
\text { Peri } \\
\text { od }\end{array}$ & $\begin{array}{l}\text { Da } \\
y-5\end{array}$ & $\begin{array}{c}\text { Germinat } \\
\text { ion \% } \\
\text { (GP) }\end{array}$ & $\begin{array}{c}\text { Root } \\
\text { length(rad } \\
\text { icle } \\
\text { length) } \\
(\mathrm{cm})\end{array}$ & $\begin{array}{c}\text { Shoot } \\
\text { length(c } \\
\text { m) }\end{array}$ & $\begin{array}{l}\text { Seedli } \\
\text { ng } \\
\text { length } \\
\text { (cm) }\end{array}$ & $\begin{array}{c}\text { Seed } \\
\text { Stamina } \\
\text { Index } \\
\text { (SSI) }\end{array}$ & $\begin{array}{l}\text { Seed } \\
\text { Vigour } \\
\text { Index } \\
\text { (SVI) }\end{array}$ & $\begin{array}{c}\text { Wet } \\
\text { weight( } \\
\text { g) }\end{array}$ & $\begin{array}{c}\text { Dry } \\
\text { weight( } \\
\text { g) }\end{array}$ & $\begin{array}{c}\% \\
\text { Moistu } \\
\text { re } \\
\text { conten } \\
\mathbf{t}\end{array}$ & $\begin{array}{c}\text { No. } \\
\text { of } \\
\text { Later } \\
\text { al } \\
\text { roots }\end{array}$ \\
\hline Oh & 6 & 60 & $1.6 \pm 0.1$ & $6.6 \pm 0.1$ & $\begin{array}{c}8.2 \pm 0 . \\
1\end{array}$ & $\begin{array}{c}4.92 \pm 0.0 \\
21\end{array}$ & $\begin{array}{c}492 \pm 2.1 \\
1\end{array}$ & $\begin{array}{c}0.36 \pm 0 \\
01\end{array}$ & $\begin{array}{c}0.16 \pm 0 \\
01\end{array}$ & 55.55 & 10 \\
\hline 1h & 9 & 90 & $5.4 \pm 0.1$ & $6.0 \pm 0.1$ & $\begin{array}{c}11.4 \pm 0 \\
.1\end{array}$ & $\begin{array}{c}10.26 \pm 0 \\
23\end{array}$ & $\begin{array}{c}1026 \pm 23 \\
.11\end{array}$ & $\begin{array}{c}0.18 \pm 0 \\
01\end{array}$ & $\begin{array}{c}0.09 \pm 0 \\
02\end{array}$ & 50.00 & 16 \\
\hline $2 h$ & 9 & 90 & $6.1 \pm 0.1$ & $6.8 \pm 0.1$ & $\begin{array}{c}12.9 \pm 0 \\
.1\end{array}$ & $\begin{array}{c}11.61 \pm 0 . \\
21\end{array}$ & $\begin{array}{c}1161 \pm 21 \\
.10\end{array}$ & $\begin{array}{c}0.20 \pm 0 \\
01\end{array}$ & $\begin{array}{c}0.10 \pm 0 \\
01\end{array}$ & 50.00 & 12 \\
\hline $3 h$ & 9 & 90 & $6.8 \pm 0.2$ & $6.1 \pm 0.2$ & $\begin{array}{c}12.9 \pm 0 \\
.2\end{array}$ & $\begin{array}{c}11.61 \pm 0 . \\
20\end{array}$ & $\begin{array}{c}1161 \pm 20 \\
.15\end{array}$ & $\begin{array}{c}0.28 \pm 0 \\
02\end{array}$ & $\begin{array}{c}0.12 \pm 0 . \\
02\end{array}$ & 57.14 & 21 \\
\hline $4 h$ & 10 & 100 & $6.4 \pm 0.1$ & $8.0 \pm 0.2$ & $\begin{array}{c}14.4 \pm 0 \\
.1\end{array}$ & $\begin{array}{c}14.40 \pm 0 \\
18\end{array}$ & $\begin{array}{c}1440 \pm 18 \\
.41\end{array}$ & $\begin{array}{c}0.38 \pm 0 \\
02\end{array}$ & $\begin{array}{c}0.18 \pm 0 \\
01\end{array}$ & 52.63 & 16 \\
\hline $5 \mathrm{~h}$ & 10 & 100 & $5.4 \pm 0.2$ & $8.2 \pm 0.1$ & $\begin{array}{c}13.6 \pm 0 \\
.1\end{array}$ & $\begin{array}{c}13.60 \pm 0 \\
24\end{array}$ & $\begin{array}{c}1360 \pm 24 \\
.36\end{array}$ & $\begin{array}{c}0.26 \pm 0 \\
01\end{array}$ & $\begin{array}{c}0.13 \pm 0 \\
02\end{array}$ & 50.00 & 12 \\
\hline 6h & 9 & 90 & $8.3 \pm 0.1$ & $9.8 \pm 0.2$ & $\begin{array}{c}18.1 \pm 0 \\
.2\end{array}$ & $\begin{array}{c}16.29 \pm 0 . \\
30\end{array}$ & $\begin{array}{c}1629 \pm 30 \\
.11\end{array}$ & $\begin{array}{c}0.49 \pm 0 \\
02\end{array}$ & $\begin{array}{c}0.23 \pm 0 \\
01\end{array}$ & 53.06 & 16 \\
\hline $12 \mathrm{~h}$ & 10 & 100 & $6.6 \pm 0.1$ & $4.8 \pm 0.2$ & $\begin{array}{c}11.4 \pm 0 \\
.2\end{array}$ & $\begin{array}{c}11.40 \pm 0 \\
20\end{array}$ & $\begin{array}{c}1140 \pm 20 \\
.14\end{array}$ & $\begin{array}{c}0.28 \pm 0 \\
01\end{array}$ & $\begin{array}{c}0.13 \pm 0 \\
01\end{array}$ & $\mathbf{5 3 . 5 7}$ & 17 \\
\hline $24 h$ & 8 & 80 & $5.2 \pm 0.1$ & $8.6 \pm 0.1$ & $\begin{array}{c}13.8 \pm 0 \\
.1\end{array}$ & $\begin{array}{c}11.04 \pm 0 . \\
10\end{array}$ & $\begin{array}{c}1104 \pm 10 \\
.25\end{array}$ & $\begin{array}{c}0.25 \pm 0 \\
01\end{array}$ & $\begin{array}{c}0.11 \pm 0 \\
01\end{array}$ & 56.00 & 17 \\
\hline
\end{tabular}

(Result=Mean \pm std $)$ 
Table 8: Treatment of Ulva lactuca on Vigna radiata L. (After 40 days)

\begin{tabular}{|c|c|c|c|c|c|c|c|c|}
\hline Concentration & $\begin{array}{l}\text { No. of } \\
\text { branches } \\
\text { per plant }\end{array}$ & $\begin{array}{l}\text { No. of } \\
\text { leaves } \\
\text { per } \\
\text { plant }\end{array}$ & $\begin{array}{l}\text { Height } \\
\text { of the } \\
\text { plant } \\
\text { (cm) }\end{array}$ & $\begin{array}{l}\text { No. of } \\
\text { flower } \\
\text { per } \\
\text { plant }\end{array}$ & $\begin{array}{l}\text { No. of } \\
\text { pod } \\
\text { per } \\
\text { plant }\end{array}$ & $\begin{array}{l}\text { No. of } \\
\text { seed } \\
\text { per } \\
\text { pod }\end{array}$ & $\begin{array}{l}\text { Length } \\
\text { of the } \\
\text { pod } \\
\text { (cm) }\end{array}$ & $\begin{array}{l}\text { Leaf } \\
\text { area } \\
\left(\mathrm{cm}^{2}\right)\end{array}$ \\
\hline $0.2 \%$ (2h) & 4 & 14 & 21 & 3 & ---- & ---- & ---- & 12.0 \\
\hline $0.5 \%$ (2h) & 4 & 14 & 22 & 2 & ---- & ---- & ---- & 10.5 \\
\hline $0.7 \%(6 h)$ & 5 & 10 & 20 & 1 & 1 & ---- & 2 & 10.0 \\
\hline $1.0 \%(12 h)$ & 4 & 10 & 23 & 1 & 1 & ---- & 2 & 9.0 \\
\hline $1.5 \%(12 \mathrm{~h})$ & 4 & 11 & 20 & ---- & 1 & ---- & 4 & 8.5 \\
\hline $2.0 \%$ (4h) & 7 & 16 & 24 & ---- & 1 & ---- & 2 & 13.0 \\
\hline Control (2h) & 5 & 14 & 15 & ---- & ---- & ---- & ---- & 12.5 \\
\hline
\end{tabular}

(Result=Mean)

Table 9: Treatment of Ulva lactuca on Vigna radiata L. (After 50 days)

\begin{tabular}{|c|c|c|c|c|c|c|c|c|}
\hline Concentration & $\begin{array}{c}\text { No. of } \\
\text { branches } \\
\text { per plant }\end{array}$ & $\begin{array}{c}\text { No. of } \\
\text { leaves } \\
\text { per } \\
\text { plant }\end{array}$ & $\begin{array}{c}\text { Height } \\
\text { of the } \\
\text { plant } \\
\text { (cm) }\end{array}$ & $\begin{array}{c}\text { No. of } \\
\text { flower } \\
\text { per } \\
\text { plant }\end{array}$ & $\begin{array}{c}\text { No. of } \\
\text { pod } \\
\text { per } \\
\text { plant }\end{array}$ & $\begin{array}{c}\text { No. of } \\
\text { seed } \\
\text { per } \\
\text { pod }\end{array}$ & $\begin{array}{c}\text { Length } \\
\text { of the } \\
\text { pod } \\
(\mathbf{c m})\end{array}$ & $\begin{array}{c}\text { Leaf } \\
\text { area } \\
\left(\mathbf{c m}^{2}\right)\end{array}$ \\
\hline $\mathbf{0 . 2 \%}$ & 4 & 14 & 22 & ---- & 2 & 6 & 7 & 12.5 \\
\hline $\mathbf{0 . 5 \%}$ & 5 & 14 & 23 & ---- & 2 & 4 & 5.5 & 11.5 \\
\hline $\mathbf{0 . 7 \%}$ & 6 & 14 & 27 & ---- & 2 & 8 & 8 & 10.0 \\
\hline $\mathbf{1 . 0 \%}$ & 5 & 11 & 23 & ---- & 2 & 2 & 5.6 & 9.0 \\
\hline $\mathbf{1 . 5 \%}$ & 4 & 11 & 20 & ---- & 1 & 3 & 6 & 8.5 \\
\hline $\mathbf{2 . 0 \%}$ & 7 & 16 & 26 & ---- & 2 & 5 & 7.6 & 13.5 \\
\hline Control & 5 & 17 & 17 & ---- & 3 & 4 & 7 & 12.5 \\
\hline
\end{tabular}

(Result=Mean)

Table 10: Treatment of Ulva lactuca on Vigna radiata L. (After 60 days)

\begin{tabular}{|c|c|c|c|c|c|c|c|c|}
\hline Concentration & $\begin{array}{c}\text { No. of } \\
\text { branches } \\
\text { per plant }\end{array}$ & $\begin{array}{c}\text { No. of } \\
\text { leaves } \\
\text { per } \\
\text { plant }\end{array}$ & $\begin{array}{c}\text { Height } \\
\text { of the } \\
\text { plant } \\
\text { (cm) }\end{array}$ & $\begin{array}{c}\text { No. of } \\
\text { flower } \\
\text { per } \\
\text { plant }\end{array}$ & $\begin{array}{c}\text { No. of } \\
\text { pod } \\
\text { per } \\
\text { plant }\end{array}$ & $\begin{array}{c}\text { No. of } \\
\text { seed } \\
\text { per } \\
\text { pod }\end{array}$ & $\begin{array}{c}\text { Length } \\
\text { of the } \\
\text { pod } \\
(\mathbf{c m})\end{array}$ & $\begin{array}{c}\text { Leaf } \\
\text { area } \\
\left(\mathbf{c m}^{2}\right)\end{array}$ \\
\hline $\mathbf{0 . 2 \%}$ & 6 & 14 & 23 & 4 & 1 & ---- & 2 & 12.5 \\
\hline $\mathbf{0 . 5 \%}$ & 6 & 14 & 24 & 2 & ---- & ---- & ---- & 11.5 \\
\hline $\mathbf{0 . 7 \%}$ & 6 & 12 & 24 & 1 & 4 & ---- & 1.2 & 10.0 \\
\hline $\mathbf{1 . 0 \%}$ & 5 & 11 & 24 & 2 & ---- & ---- & ---- & 9.0 \\
\hline $\mathbf{1 . 5 \%}$ & 5 & 11 & 18 & 1 & ---- & ---- & ---- & 8.5 \\
\hline $\mathbf{2 . 0 \%}$ & 7 & 17 & 28 & 3 & 1 & ---- & 0.8 & 13.5 \\
\hline Control & 7 & 15 & 17 & 2 & ---- & ---- & ---- & 12.5 \\
\hline
\end{tabular}

(Result=Mean) 
Table 11: Physicochemical analysis of Ulva lactuca L. extract at different concentration

\begin{tabular}{|l|l|l|l|l|l|l|}
\hline \multicolumn{1}{|c|}{ Name } & $\begin{array}{l}\text { Salt Conc. } \\
(\mathbf{p p m})\end{array}$ & $\mathbf{P}^{\mathrm{H}}$ & $\begin{array}{l}\text { ORP } \\
(\mathbf{m V})\end{array}$ & $\begin{array}{l}\text { COND } \\
(\boldsymbol{\mu s})\end{array}$ & $\begin{array}{l}\text { TDS } \\
(\mathbf{p p m})\end{array}$ & $\begin{array}{l}\text { Temp } \\
\left({ }^{\mathbf{6}} \mathbf{C}\right)\end{array}$ \\
\hline Ulva lactuca (0.2\%) & 125 & 8.96 & -97.6 & 260 & 168 & 26.6 \\
\hline Ulva lactuca (0.5\%) & 167 & 7.70 & -20.6 & 346 & 227 & 26.3 \\
\hline Ulva lactuca (0.7\%) & 150 & 7.79 & -27.6 & 302 & 198 & 26.2 \\
\hline Ulva lactuc (1.0\%) & 187 & 7.68 & -19.8 & 383 & 252 & 26.3 \\
\hline Ulva lactuca (1.5\%) & 216 & 7.94 & -34.4 & 442 & 289 & 26.8 \\
\hline Ulva lactuca (2.0\%) & 230 & 7.62 & -20.7 & 474 & 312 & 27.1 \\
\hline Control & ---- & 7.0 & ---- & 0 & ---- & ---- \\
\hline
\end{tabular}

3.3 Biochemical properties of Vigna radiata $\mathrm{L}$. treated with seaweed extract Ulva lactuca

Maximum concentration of $0.2371 \mathrm{mg} / \mathrm{g}$ dry weight of chlorophyll a, $0.5491 \mathrm{mg} / \mathrm{gm}$ dry weight of chlorophyll $\mathrm{b}$ and $0.5451 \mathrm{mg} / \mathrm{gm}$ dry weight of total chlorophyll was recorded at $0.5 \%$ of $U$. lactuca seaweed extract treatment (Figure 1). Their decreases more than 43\%, 54.87\% and $53.47 \%$ respectively, when compared to control. Carotenoid also continue decreases amount of all seaweed extract concentration (Figure 2). The maximum concentration of $3.8475 \mathrm{mg} / \mathrm{gm}$ dry weight of protein was recorded at $1.5 \%$ seaweed extract in $U$. lactuca (Figure 3 ). A maximum concentration of $7.9677 \mu \mathrm{g} / \mathrm{ml}$ dry weight of total soluble sugar was recorded at $0.2 \% \quad U$. lactuca seaweed extract treatment (Figure 4). In total soluble sugar amount of concentration was high compared to control. Maximum concentration of $11.4245 \mu \mathrm{g} / \mathrm{ml}$ dry weight of reducing sugar was recorded at $0.7 \%$ of $U$. lactuca seaweed extract treatment (Figure 5). Their decrease $62.72 \%$ compared to control.

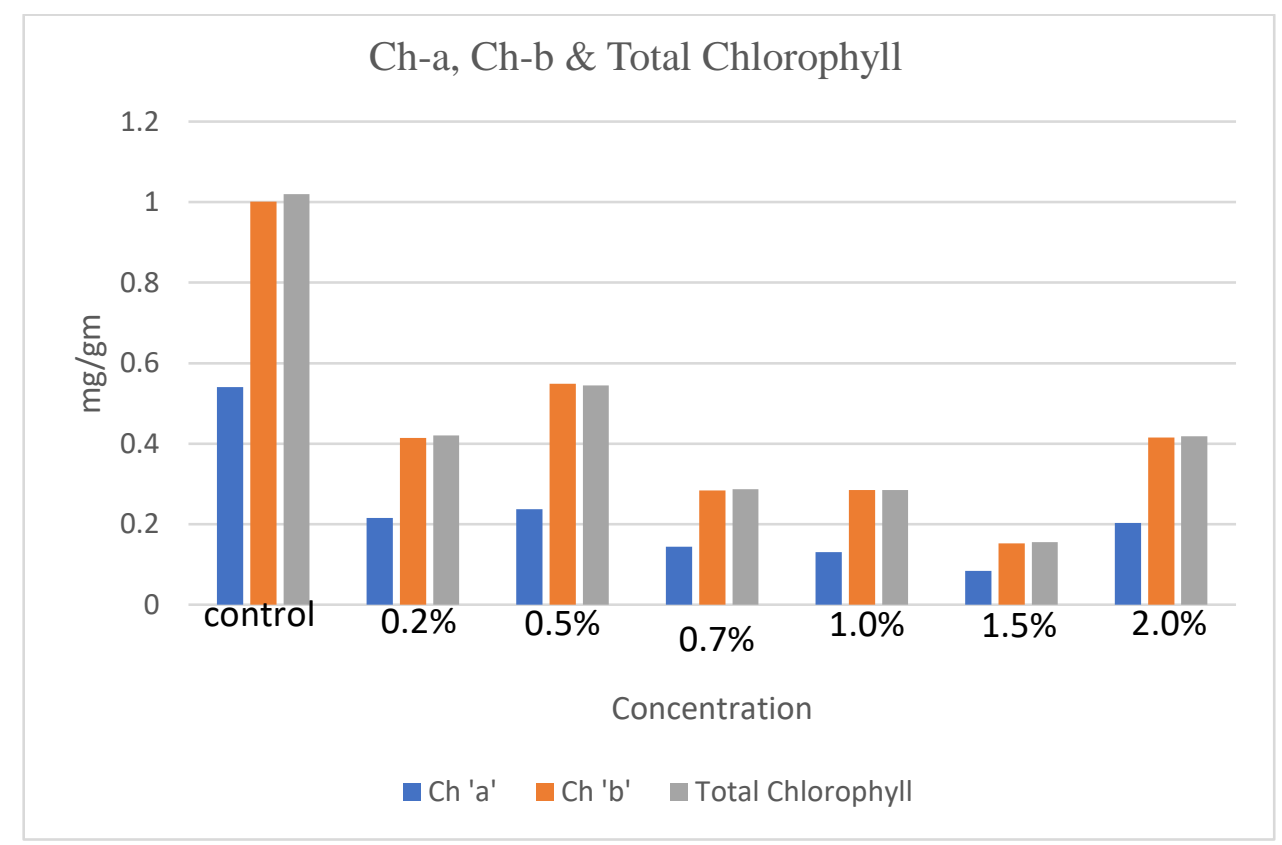

Fig.1: Effect of Ulva lactuca Seaweed biostimulant on the Chlorophyll-a, chlorophyll-b \& Total chlorophyll content of Vigna radiata $L$. 


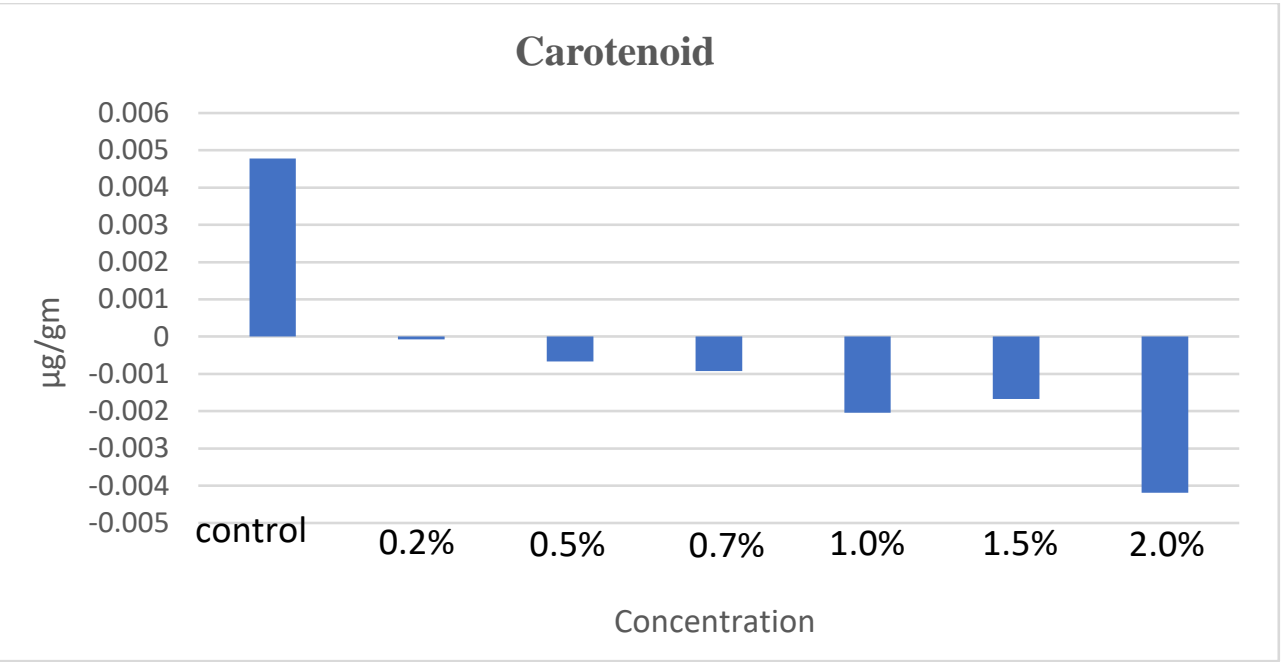

Fig.2: Effect of Ulva lactuca Seaweed biostimulant on the Carotenoid content of Vigna radiata L.

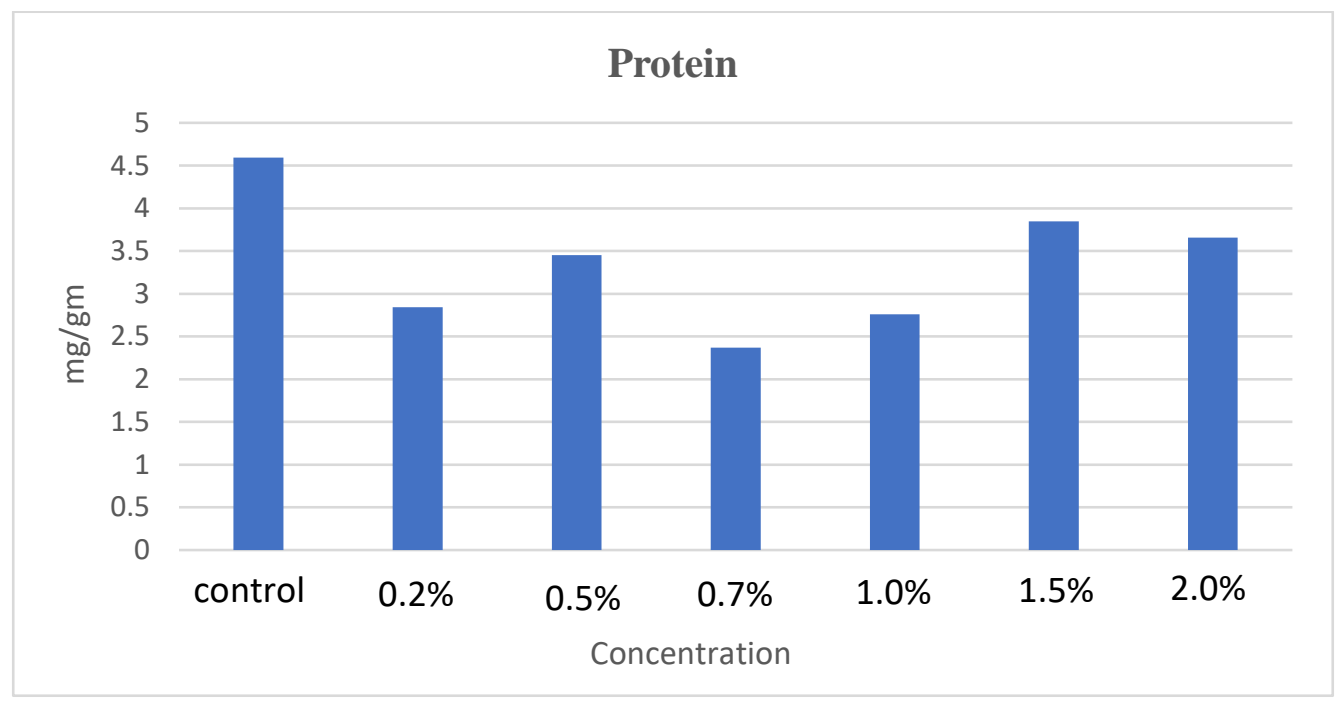

Fig.3: Effect of Ulva lactuca Seaweed biostimulant on the Protein content of Vigna radiata L.

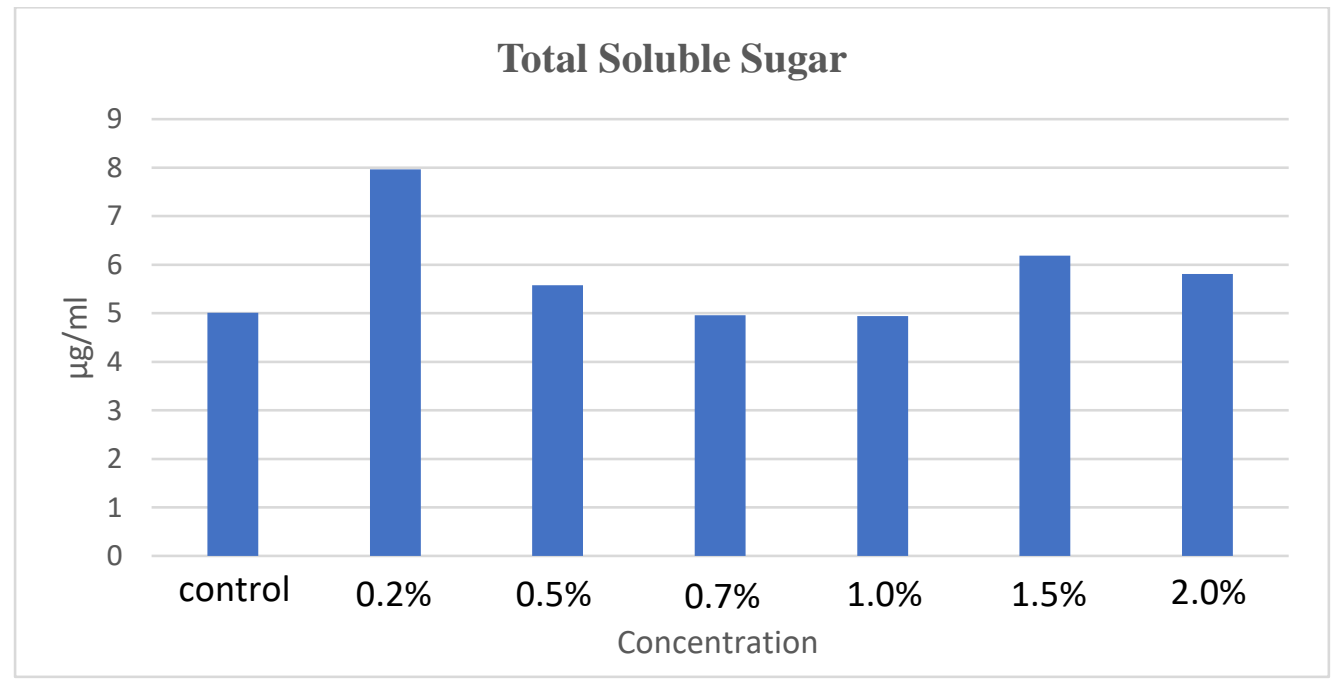

Fig.4: Effect of Ulva lactuca Seaweed biostimulant on the Total Soluble Sugar content of Vigna radiata L. 


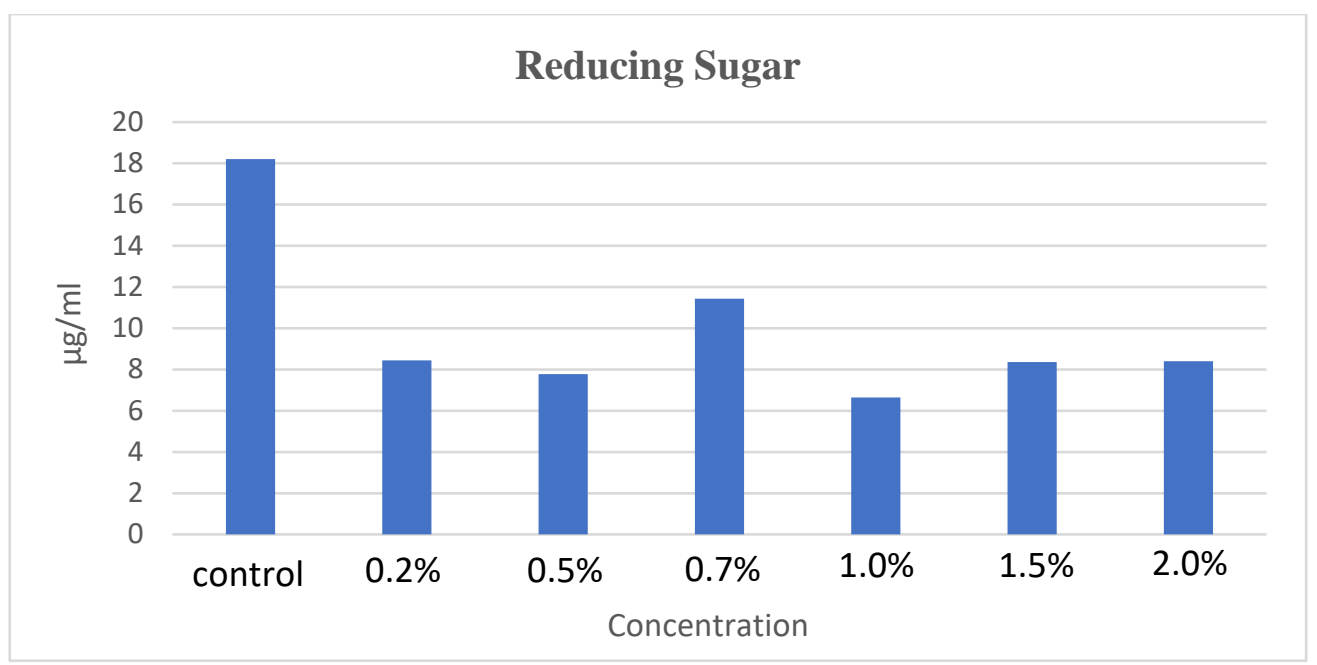

Fig.5: Effect of Ulva lactuca Seaweed biostimulant on the Reducing Sugar content of Vigna radiata L.

\section{DISCUSSION}

The Vigna radiata L. seeds soaked with higher concentrations of the seaweed biostimulant showed higher rates of germination, while the lower concentrations of the extracts inhibited the germination. The value of seaweed as fertilizer is not from the nitrogen, phosphorous, potash and organic matter but from trace element and metabolites similar to plants growth regulators (Booth, 1969). Seaweed liquid fertilizer was found superior than chemical fertilizer because of the presence of high level of organic matter (Aitken and Senn, 1965). The increased germination percentage at high concentrations may be due to the presence of some growth promoting substances such as IAA and IBA, Gibberellins (A\&B), cytokinins, micronutrients ( $\mathrm{Fe}, \mathrm{Cu}, \mathrm{Zn}, \mathrm{Co}, \mathrm{Mo}, \mathrm{Mn}, \mathrm{Ni}$ ), vitamins and amino acids (Challen and Hemingway, 1965). The increased seed germination percentage at high concentrations may be due to the presence of growth promoting substances (Jennings and Yulloch, 1965). The present study highlights the efficiency of Seaweed biostimulants obtained from the green seaweed, Ulva lactuca. Similar results were recorded in Cajanus cajan red gram (Kumar, Mohan, Murugeswari and Muthusamy, 1993), Oryza sativa (Kumar, 2009) and Vigna mungo (Ganapathy, Balamurugan, thinakaran and Sivakumar, 2013). Reported that the presence of plant growth regulators, trace elements, vitamins and macronutrients in the seaweed liquid fertilizer enhance the growth of root length and shoot length of Vigna mungo (Challen and Hemingway, 1965). The present investigation is in agreement with the earlier studies (Xavier and Jesudas, 2007). The lower concentrations showed a decreasing trend. Some similar results were recorded in Pedina which induced maximum seedling growth at lower concentrations in $C$. cajan and Vigna radiata. Reported similar findings with Hypneamusciformis, Spatoglossum asperum, Stoechospermum marginatum and Sargassum on the growth of crops such as green chillies, turnips and pineapple (Dhargalkar and Untawale, 1983). Additionally, it is known that higher concentrations of $(1.0 \%)$ the Ulva lactuca liquid extract can inhibit the germination of mung bean (Castellanos-Barriga, 2017). In this study, total chlorophyll contents highest in $0.5 \%$ concentration in $U$. lactuca seaweed biostimulants and lowest in $1.5 \%$ seaweed biostimulants in U. lactuca. Protein, total soluble sugar and reducing sugar was highest in $1.5 \%, 0.2 \%$ and $0.7 \%$ concentrations respectively, and lowest in $0.7 \%$ and $1.0 \%$ respectively. Vigna catajung when treated with $10 \%$ Caulerpa racemose extract contained highest protein and amino acid contents (Anantharaj and Venkatesalu, 2001). The amount of protein content was found highest the shoot system under the treatment of Enteromorpha and Jania extract (El-Sheekh and El-Saied, 1999).

\section{CONCLUSION}

The results obtained in this experiment showed this presoaking experiment seed with seaweed extract for improve and increase seed germination and plant growth of selected plant of Vigna radiata L. the study showed that there is significant effect of treatments with seaweed extract from Ulva lactuca on bio-chemical parameter of chlorophyll a, b \& total, carotenoid, protein, total soluble sugar and reducing sugar. The seed priming is a new and unique method for improvement of healthy and fast seed germination. Seaweed biostimulants as a Bio-primer material and this Bio-priming method is a organic, very easy, very cheap, easily available and eco-friendly. Based on this experiment, green algae of Ulva lactuca on giving a result of in a plant of Vigna radiata L. higher growth are 
observed in $1.5 \%$ and $2.0 \%$ concentration, Biochemical parameter are observed in lower concentration for better result are observed. The possibility of the existence of minerals and polysaccharides in algal extracts auxin-like activity is high and could be responsible for the effect on growth parameters.

\section{ACKNOWLEDGMENT}

I am very thankful to my Guide \& Head, Botany Department, Sir P. P. Institute of Science, Maharaja Krishnakumarsinhji Bhavnagar University, Bhavnagar, Gujarat for moral support and thankful to Dr. Bharatsingh Gohil, Department of Life sciences, Bhavnagar for providing necessary facilities to carry out this work. I am also thankful Education Department, Gujarat state for providing SHODH (ScHeme of Developing High quality research) scholarship for financial assistance.

\section{REFERENCES}

[1] Challen SB, Hemingway JG (1965). Growth of higher plants in response to feeding with seaweed extract. Proc Fifth Int. Seaweed symp. 5: 359-367.

[2] Jennings, D.L. and B.M.M. Tulloch (1965). Growth promoting hormones in seaweeds. J. Exp. Bot., 16: 329.

[3] Venkataraman Kumar V, Mohan VR, Murugeswari R, Muthusamy M (1993). Effect of crude and commercial seaweed extracts on seed germination and seedling growth in green gram and black gram. Seaweed Res. Utiln. 16: $23-$ 27.

[4] Asir Selin Kumar (2009). Influence of seagrass Syringodiumisoetifolium extract on germination and growth of Oryza sativa. J. Ecotoxicol. Environ. Onit., 19 (2): 157 162.

[5] Sobithabai, R., J. Irene Wilsey and R. Asir Selin Kumar (2005). Influence of seagrass liquid fertilizer on cotyledons in cyamosistetragonoloba Taub. Seaweed Res. Utiln., 27(1\&2): 155-157.

[6] Sivasankari, S., M. Chandrasekaran, M.Kannathasan and V. Venkatesalu (2006). Studies on the biochemical constituents of Vigna radiata Linn. Treated with seaweed liquid fertilizer. Seaweed Res. Utiln., 28(1): 151-158.

[7] Sahaya Anthony Xavier, G. and L. Louis Jesudas (2007). Effect of seaweed extract on cluster bean. Seaweed Res. Utiln., 29(1\&2): 85-87.

[8] Dhargalkar,V.K., Untawale, A.G., (1983). Some observations of the effect of SLF, on higher plants. Indian J. Mar. Sci. 12, 210-214.

[9] Ganapathy Selvam, G., Balamurugan. M., Thinakaran, T. and Sivakumar, K., (2013). Developmental changes in the germination, growth and chlorophyllase activity of Vigna mungo L. using seaweed extract of Ulva raticulataForsskal. IRJP., 4(1): 252-254.

[10] Mishra P. and Dash D. (2014). Rejuvenation of Biofertiliser for Sustainable Agriculture Economic
Development (SAED), Consilience: The Journal of Sustainable Development., 11:41-61.

[11] Fleurence J. (1999). Seaweed proteins: biochemical nutritional aspects and potential uses. Trends in Food Science and Technology. 10: 25-28.

[12] R. V. Patel, K. Y. Pandaya, R.T. Jasrai and N. Brahmbhatt (2017). Effect of hydro priming and biopriming on seed germination of Brinjal and Tomato seed. Research Journal of Agriculture and Forestry Sciences Vol. 5(6): 1-14.

[13] Shridhar S, Rengasamy R (2010). Effect of seaweed liquid fertilizer on the growth, biochemical constituents and yield of Tagetes erecta under field trial. J. Phytol 2(6): 61-68.

[14] Anantharaj, M. and Venkatesalu, V. (2001). Effect of seaweed liquid fertilizer on Vigna calajung. Seaweed Res. Utiln., 23: 33-39.

[15] S. Sridhar and R. Rengasamy (2011). Effect of seaweed liquid fertilizer on growth, pigment concentration and yield of Amaranthus roxburghinus and Amaranthus tricolor under field trial. IJCR 3(7): 131-134. 